\title{
Oclusão Percutânea de Comunicação Interatrial Tipo Ostium Secundum com Prótese Memopart ${ }^{\circledR}$
}

Jorge Luis Haddad ${ }^{1}$, Gustavo Caires Novaes², Rafael Brolio Pavão ${ }^{3}$, Andre Vannucchi Badran ${ }^{4}$, Daniel Conterno Lemos ${ }^{5}$, Igor Matos Lago ${ }^{6}$, Moyses de Oliveira Lima-Filho ${ }^{7}$, Geraldo Luiz de Figueiredo ${ }^{8}$, Paulo Henrique Manso ${ }^{9}$, Walter Villela de Andrade Vicente ${ }^{10}$, Luis Gustavo Gali ${ }^{11}$, Minna Moreira Dias Romano ${ }^{12}$, J. Antonio Marin-Neto ${ }^{13}$

\section{RESUMO}

Introdução: Os autores relatam a experiência inicial da oclusão da comunicação interatrial ostium secundum (CIA) com a utilização da prótese Memopart ${ }^{\circledR}$ (Shanghai Shape Memory Alloy Co Ltd, Shanghai, China). Métodos: Estudo prospectivo observacional, no qual uma série de pacientes portadores de defeitos com significativa repercussão hemodinâmica e características anatômicas favoráveis ao implante foi submetida à oclusão percutânea de CIA. O procedimento foi realizado por via femoral percutânea, pela técnica habitual. O período de seguimento foi de 10,3 \pm 5 meses, com controles clínicos e ecocardiográficos 24 horas (ou antes da alta hospitalar), 1, 3, 6 e 12 meses após o implante. Resultados: No período de fevereiro de 2012 a abril de 2013, foram submetidos à oclusão percutânea de $\mathrm{CIA} 21$ pacientes, sendo 16 do sexo feminino, com idade média de 33,1 $\pm 18,7$ anos. O diâmetro médio do defeito foi de 19,04 $\pm 6,25 \mathrm{~mm}$ e o tamanho da prótese foi de $21,42 \pm 6,73 \mathrm{~mm}$ ( 8 a $34 \mathrm{~mm}$ ). O implante foi realizado com êxito em todos os casos, verificando-se oclusão total no controle antes da alta hospitalar. No seguimento, todos os pacientes estiveram assintomáticos e comprovou-se a persistência da oclusão total do defeito. Não houve mortalidade e nem outras complicações na série. Conclusões: A oclusão percutânea da CIA utilizando-se prótese Memopart ${ }^{\circledR}$

\section{ABSTRACT}

\section{Percutaneous Closure of Ostium Secundum Atrial Septal Defect Using the Memopart ${ }^{\mathrm{TM}}$ Occlusion Device}

Background: The authors report their initial experience with the Memopart ${ }^{\top M}$ device (Shanghai Shape Memory Alloy Co Ltd, Shanghai, China) for the occlusion of secundum atrial septal defect (ASD). Methods: This was a prospective observational study of a series of patients undergoing percutaneous occlusion of ASD with right ventricle volume overload and favorable anatomic characteristics. The procedure was performed by percutaneous femoral approach. The mean follow-up was 10.3 \pm 5 months, with clinical and 24-hour echocardiographic evaluations (or before hospital discharge), 1, 3, 6, and 12 months after implantation. Results: From February/2012 to April/2013, 21 patients, 16 females, mean age $33.1 \pm 18.7$ years, were submitted to percutaneous occlusion of an ASD. The average diameter of the defect was $19.04 \pm 6.25 \mathrm{~mm}$ and the device size was $21.42 \pm 6.73 \mathrm{~mm}$ (8 to $34 \mathrm{~mm}$ ). Total occlusion of the defect was observed in all cases before hospital discharge. During follow-up, all patients were asymptomatic and without residual shunt. There was no deaths or any other complications in the series. Conclusions: The percutaneous closure
1 Cardiologista intervencionista do Hospital das Clínicas da Faculdade de Medicina de Ribeirão Preto da Universidade de São Paulo. Ribeirão Preto, SP, Brasil.

${ }^{2}$ Residente em Cardiologia Intervencionista Pediátrica do Hospital das Clínicas da Faculdade de Medicina de Ribeirão Preto da Universidade de São Paulo. Ribeirão Preto, SP, Brasil.

${ }^{3}$ Residente em Cardiologia Intervencionista do Hospital das Clínicas da Faculdade de Medicina de Ribeirão Preto da Universidade de São Paulo. Ribeirão Preto, SP, Brasil.

${ }^{4}$ Residente em Cardiologia Intervencionista do Hospital das Clínicas da Faculdade de Medicina de Ribeirão Preto da Universidade de São Paulo. Ribeirão Preto, SP, Brasil.

${ }^{5}$ Cardiologista intervencionista do Hospital das Clínicas da Faculdade de Medicina de Ribeirão Preto da Universidade de São Paulo. Ribeirão Preto, SP, Brasil.

${ }^{6}$ Cardiologista intervencionista do Hospital das Clínicas da Faculdade de Medicina de Ribeirão Preto da Universidade de São Paulo. Ribeirão Preto, SP, Brasil.

Doutor. Cardiologista intervencionista do Hospital das Clínicas da Faculdade de Medicina de Ribeirão Preto da Universidade de São Paulo. Ribeirão Preto, SP, Brasil.

\footnotetext{
${ }^{8}$ Cardiologista intervencionista do Hospital das Clínicas da Faculdade de Medicina de Ribeirão Preto da Universidade de São Paulo. Ribeirão Preto, SP, Brasil.

${ }^{9}$ Doutor. Cardiologista pediátrico da Faculdade de Medicina de Ribeirão Preto da Universidade de São Paulo. Ribeirão Preto, SP, Brasil. ${ }^{10}$ Livre-Docente. Cirurgião cardiovascular da Faculdade de Medicina de Ribeirão Preto da Universidade de São Paulo. Ribeirão Preto, SP, Brasil. ${ }^{11}$ Médico do Setor de Ecocardiografia do Hospital das Clínicas da Faculdade de Medicina de Ribeirão Preto da Universidade de São Paulo. Ribeirão Preto, SP, Brasil.

12 Doutora. Cardiologista e docente da Faculdade de Medicina de Ribeirão Preto da Universidade de São Paulo. Ribeirão Preto, SP, Brasil. ${ }^{13}$ Livre-docente. Diretor da Unidade de Hemodinâmica e Cardiologia Intervencionista do Hospital das Clínicas da Faculdade de Medicina de Ribeirão Preto da Universidade de São Paulo. Ribeirão Preto, SP, Brasil.

Correspondência: Jorge Luis Haddad. Campus Universitário Monte Alegre - Avenida Bandeirantes, 3.900 - Monte Alegre - Ribeirão Preto, SP, Brasil - CEP 14049-900

E-mail: jl.haddad@yahoo.com.br
}

Recebido em: 4/9/2013 • Aceito em: 18/11/2013 
é um procedimento eficaz e seguro, dentro dos limites desta investigação. O implante da prótese é simples e apresenta alto índice de oclusão imediata, inclusive de defeitos de grandes dimensões.

DESCRITORES: Comunicação interatrial. Dispositivo para oclusão septal. Cardiopatias congênitas. Cateterismo cardíaco.

A comunicação interatrial do tipo ostium secundum (CIA), localizada na fossa oval, constitui aproximadamente $80 \%$ dos defeitos septais interatriais. ${ }^{1}$ A oclusão percutânea deve ser realizada, nos casos em que a anatomia é favorável, na vigência de repercussão hemodinâmica (indicação classe I, nível de evidência B), na associação com síndrome de platipneia ortodeoxia (indicação classe Ila, nível de evidência B) ou quando houver antecedente de embolia paradoxal (indicação classe Ila, nível de evidência C). ${ }^{2}$ Aproximadamente 85\% das $\mathrm{CIA}$ apresentam os fatores estruturais fundamentais que determinam uma anatomia propícia à oclusão percutânea, que são: diâmetro estirado do defeito $<42$ mm, que é o diâmetro máximo das próteses disponíveis, e bordos com dimensões $\geq 5 \mathrm{~mm}$, com exceção do bordo aórtico ou anterossuperior.

Os dispositivos mais utilizados para oclusão percutânea são os de duplo disco de nitinol, que são autocentráveis, recuperáveis e reposicionáveis, de fácil manejo e apresentam altas taxas de sucesso na oclusão das CIA. Essas próteses estão constituídas por numerosos filamentos de nitinol (liga de níquel e titânio), soldados em suas extremidades e moldados para formar uma cintura central, que atua como um stent no orifício da comunicação de dois discos, um esquerdo maior e outro direito menor. As diferentes próteses desse tipo apresentam pequenas variações em relação à espessura dos fios de nitinol, aos retalhos inseridos no interior dos discos, às dimensões dos mesmos em relação à cintura central e ao sistema de fixação do dispositivo ao cabo de entrega.

O objetivo deste trabalho foi relatar os resultados obtidos em série prospectiva de pacientes submetidos à oclusão percutânea de $\mathrm{CIA}$ com a prótese Memopart ${ }^{\circledR}$ (Shanghai Shape Memory Alloy Co. Ltd., Shanghai, China).

\section{MÉTODOS}

\section{Desenho do estudo}

Estudo prospectivo de coorte, longitudinal e observacional realizado em um único centro, no período de fevereiro de 2012 a abril de 2013, com pacientes submetidos à oclusão percutânea de CIA utilizando-se prótese Memopart ${ }^{\circledR}$. O estudo foi realizado no Laboratório de Hemodinâmica e Cardiologia Intervencionista do Hospital das Clínicas da Faculdade de Medicina de Ribeirão Preto da Universidade de São Paulo (HCFMRP- of ASD using a Memopart ${ }^{\top M}$ device is an effective and safe procedure within the limits of this investigation. The device is user-friendly and has a high rate of immediate occlusion, even in large defects.

KEYWORDS: Heart septal defects, atrial. Septal occluder device. Heart defects, congenital. Cardiac catheterization.

USP) e obteve aprovação do Comitê de Ética da instituição. Todos os pacientes ou seus responsáveis legais assinaram um termo de consentimento após esclarecimento sobre os propósitos do estudo.

\section{Critérios de inclusão}

Participaram do estudo portadores de CIA referidos ao HCFMRP-USP com as seguintes característica clínicas, anatômicas e hemodinâmicas: peso > 25 kg; dilatação de câmaras cardíacas direitas visualizadas durante avaliação ecocardiográfica; relação QP/QS $>1,5$; pressão arterial pulmonar $\leq 2 / 3$ do nível sistêmico; resistência vascular pulmonar $<5 \mathrm{UW}$; tamanho do diâmetro do defeito $<42 \mathrm{~mm}$; bordos do defeito $>5 \mathrm{~mm}$, exceto o retroaórtico; assinatura do termo de consentimento livre e esclarecido aprovado pela Comissão de Ética institucional.

\section{Critérios de exclusão}

Foram excluídos pacientes com associação de outras cardiopatias congênitas ou adquiridas com indicação de correção cirúrgica; com bordos dos defeitos septais finos, hipermóveis ou deficientes; com infecção ativa de qualquer natureza ou processo infecioso no mês prévio; portadores de síndrome de hipercoagulabilidade; com contraindicação de medicação antiplaquetária.

\section{Prótese}

A prótese Memopart ${ }^{\circledR}$ (Figura 1) é um dispositivo manufaturado com fios de nitinol, uma liga metálica de níquel e titânio na proporção, em relação ao peso atômico, de 55 e 45\%, respectivamente. O conjunto dos fios é soldado nos extremos, constituindo um feixe, com pinos em ambos os extremos. O feixe é posteriormente moldado, em alta temperatura, na forma de um duplo disco de tamanhos diferentes, com uma cintura no meio, a qual atua como mecanismo centralizador e posiciona-se no defeito como um stent. O pino do disco menor, que é o direito, apresenta um sistema de rosca, que é parafusado no cabo liberador. A prótese é fixada no cabo liberador e esticada, para ser introduzida no carregador e transferida para a bainha de entrega. Devido à memória térmica do nitinol, o dispositivo, quando exteriorizado, readquire a forma original.

O diâmetro do disco central determina o tamanho do dispositivo, que varia de 6 a $42 \mathrm{~mm}$, com incremento de $1 \mathrm{~mm}$, entre os tamanhos de 6 a $20 \mathrm{~mm}$, e 
de $2 \mathrm{~mm}$, entre os tamanhos 22 a $42 \mathrm{~mm}$. As características técnicas das próteses e bainhas liberadoras são expostas na Tabela 1.

\section{Procedimento}

Os procedimentos foram realizados por abordagem em veia femoral, sob anestesia geral, e com ecocardiografia transesofágica. Foi administrada heparina não fracionada na dose de $100 \mathrm{UI} / \mathrm{kg}$ e realizada profilaxia antimicrobiana com cefalosporina de primeira geração. O tamanho da CIA foi determinado com cateter-balão por técnica estática, e o diâmetro no qual houve a cessação do fluxo ao color Doppler foi mensurado por radioscopia e ecocardiografia. A técnica de implante das próteses de duplo disco de nitinol já foi exaustivamente descrita por outros autores. ${ }^{3,4}$ Sucintamente, a prótese é mergulhada em soro fisiológico, para retirar o ar da malha e dos retalhos; parafusa-se o pino do disco direito no cabo liberador; a prótese, então, é introduzida no carregador e transferida para a bainha de entrega, previamente posicionada na veia pulmonar superior esquerda. O disco esquerdo é exteriorizado no átrio esquerdo, próximo à desembocadura da veia pulmonar, retirando-se a bainha de entrega da veia pulmonar e tracionando-se, posteriormente, o conjunto, até se ancorar o disco no septo interatrial. Mantendo-se o cabo liberador fixo, a bainha é retirada, até a exteriorização do disco direito. Comprovando-se a posição adequada do dispositivo nos três cortes ecocardiográficos básicos (eixo curto, bicaval e 4 câmaras), bem como a ausência de fluxo residual significativo, a prótese é liberada, desparafusando-se o cabo liberador por rotação horária (Figuras 2 e 3).

Diversas variações dessa técnica básica foram, às vezes, implementadas, para um adequado posicionamento da prótese, de acordo com as diferentes características anatômicas da CIA. Entre elas estão a abertura parcial da cintura, antes de ancorar o disco esquerdo; a abordagem inicial desde a veia pulmonar superior direita ou esquerda; e a exteriorização parcial do disco esquerdo na veia pulmonar, seguida da retirada da bainha até o átrio direito, para impactar primeiramente o disco direito no septo interatrial. ${ }^{5,6}$

O sucesso do procedimento foi definido quando a prótese foi implantada com posicionamento adequado e ausência ou shunt residual $<2 \mathrm{~mm}$ no ecocardiograma transesofágico realizado durante o procedimento.

\section{Seguimento}

Os pacientes foram avaliados clinicamente e com ecocardiograma transtorácico antes da alta hospitalar e após 1, 3, 6 e 12 meses. Foram medicados, nesse período, com ácido acetilsalicílico em dose única diária de $5 \mathrm{mg} / \mathrm{kg}$ (dose máxima de $100 \mathrm{mg} / \mathrm{dia}$ ), durante 6 meses. Nos pacientes adultos, foi associado clopidogrel na dose de $75 \mathrm{mg} /$ dia por 6 meses. Foi prescrita profilaxia antibacteriana, na eventualidade de procedimentos cirúrgicos ou intervencionistas, durante os primeiros 6 meses de evolução.

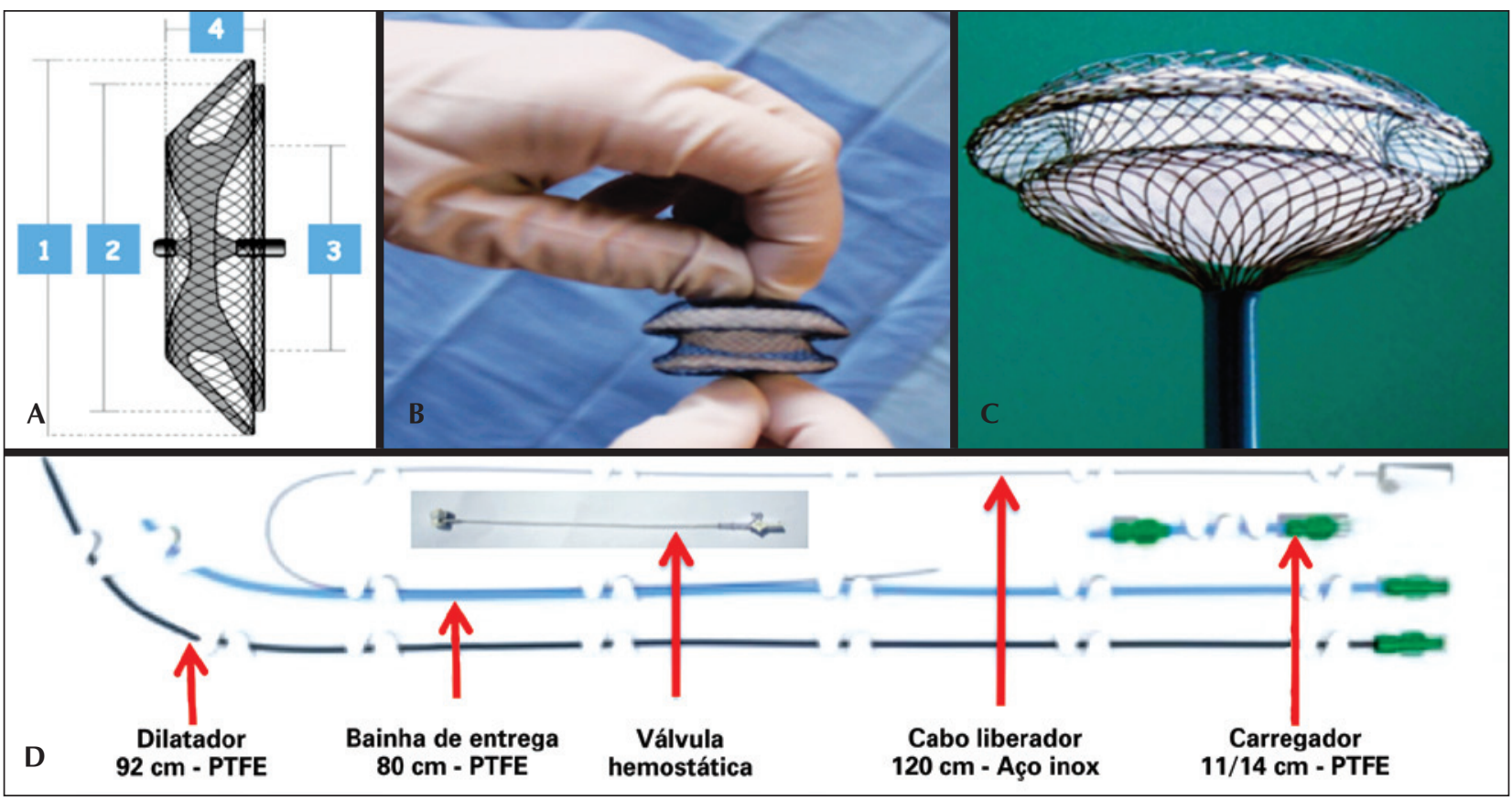

Figura 1. Prótese e conjunto de entrega e liberação. (A) Esquema da prótese: (1) diâmetro do disco esquerdo; (2) diâmetro do disco direito; (3) diâmetro do disco central; (4) espessura total do dispositivo. (B) Prótese discretamente tracionada pelos extremos proximal e distal, mostrando ambos os discos esquerdo superior e direito inferior, além do disco central. (C) Prótese carregada no sistema de entrega, parcialmente exteriorizada com o disco direito ainda não totalmente exposto. (D) Os diversos componentes do sistema de entrega e liberação. 
TABELA 1

Parâmetros clínicos, ecocardiográficos e hemodinâmicos dos pacientes

\begin{tabular}{|c|c|c|c|c|c|c|c|c|c|}
\hline \multirow[b]{2}{*}{ Paciente } & \multirow{2}{*}{$\begin{array}{l}\text { Idade } \\
\text { (anos) }\end{array}$} & \multirow{2}{*}{$\begin{array}{l}\text { Peso } \\
\text { (kg) }\end{array}$} & \multirow[b]{2}{*}{ Sexo } & \multicolumn{2}{|c|}{ Diâmetro da comunicação interatrial (mm) } & \multirow[b]{2}{*}{ QP/QS } & \multirow{2}{*}{$\begin{array}{l}\text { Prótese } \\
(\mathbf{m m})\end{array}$} & \multicolumn{2}{|c|}{ Fluxo residual } \\
\hline & & & & Ecocardiograma transesofágico & Cateterismo cardíaco & & & Imediato & 24 horas \\
\hline 1 & 8 & 35 & $\mathrm{~F}$ & 7 & 7 & 1,5 & 8 & Não & Não \\
\hline 2 & 45 & 67 & $\mathrm{~F}$ & 8 & 14 & 1,9 & 16 & Não & Não \\
\hline 3 & 35 & 55 & $\mathrm{~F}$ & 18 & 23 & 3,3 & 24 & Mínimo & Não \\
\hline 4 & 3 & 16 & $\mathrm{~F}$ & 14 & 18 & 4,8 & 20 & Não & Não \\
\hline 5 & 15 & 83 & $\mathrm{~F}$ & 13 & 16 & 2,6 & 18 & Não & Não \\
\hline 6 & 20 & 87 & $M$ & 12 & 11 & 1,7 & 12 & Não & Não \\
\hline 7 & 75 & 52 & $\mathrm{~F}$ & 6 & 14 & 1,9 & 16 & Não & Não \\
\hline 8 & 37 & 94 & $F$ & 24 & 32 & 1,9 & 34 & Não & Não \\
\hline 9 & 47 & 66 & $\mathrm{~F}$ & 15 & 17 & 3,0 & 20 & Não & Não \\
\hline 10 & 9 & 29 & $M$ & 10 & 17 & 2,9 & 18 & Não & Não \\
\hline 11 & 54 & 54 & $M$ & 20 & 26 & 4,6 & 30 & Não & Não \\
\hline 12 & 41 & 69 & $\mathrm{~F}$ & 15 & 18 & 2,7 & 22 & Não & Não \\
\hline 13 & 57 & 79 & $\mathrm{~F}$ & 17 & 21 & 1,9 & 22 & Não & Não \\
\hline 14 & 9 & 25 & $\mathrm{~F}$ & 16 & 18 & 1,8 & 24 & Não & Não \\
\hline 15 & 27 & 55 & $\mathrm{~F}$ & 15 & 24 & 3,0 & 26 & Não & Não \\
\hline 16 & 29 & 78 & $\mathrm{~F}$ & 20 & 24 & 2,6 & 28 & Mínimo & Não \\
\hline 17 & 39 & 105 & $\mathrm{~F}$ & 10 & 18 & 2,0 & 20 & Não & Não \\
\hline 18 & 44 & 84 & $\mathrm{~F}$ & 21 & 27 & 2,5 & 30 & Mínimo & Não \\
\hline 19 & 51 & 60 & $F$ & 10 & 10 & 1,6 & 12 & Não & Não \\
\hline 20 & 12 & 31 & $M$ & 13 & 16 & 1,7 & 18 & Mínimo & Não \\
\hline 21 & 38 & 99 & $M$ & 24 & 29 & 2,7 & 32 & Mínimo & Não \\
\hline
\end{tabular}

\section{Análise estatística}

As variáveis contínuas foram descritas com valores máximos e mínimos, assim como a média e o desvio padrão. As variáveis categóricas foram descritas como frequências ou percentagens.

\section{RESULTADOS}

Foram submetidos ao procedimento 21 pacientes, sendo 16 do sexo feminino, com idade média de 33,1 $\pm 18,7$ anos e peso de $63,0 \pm 24,81 \mathrm{~kg}$. A análise ecocardiográfica transesofágica demonstrou defeitos septais únicos em todos os pacientes, com diâmetros máximos entre 7 e $24 \mathrm{~mm}(14,66 \pm 5,1 \mathrm{~mm})$. Todos os pacientes tratados apresentaram bordos $>5 \mathrm{~mm}$, mas em oito pacientes houve bordo aórtico deficiente ou ausente. Os parâmetros clínicos, ecocardiogáficos e hemodinâmicos estão expostos na Tabela 1.

O cateterismo cardíaco revelou relação média entre os fluxos pulmonar e sistêmico (QP/QS) de 2,47 $\pm 1,14$, pressão sistólica da artéria pulmonar (PSAP) de $35,2 \pm 9,5 \mathrm{mmHg}$ e resistência arteriolar pulmonar de $0,9 \pm 0,5$ UW. O diâmetro da CIA medido por cateter balão variou entre 7 e 32 mm (19,04 \pm 6,25 mm) e determinou a seleção da prótese, sendo utilizados
21 dispositivos de tamanho entre 8 e $34 \mathrm{~mm}(21,42$ $\pm 6,73 \mathrm{~mm}$ ).

O implante foi possível em todos os pacientes. Em dois pacientes, houve a necessidade de posicionamento da bainha na veia pulmonar superior direita; em um paciente, a porção distal do disco esquerdo foi liberada dentro da veia pulmonar, sendo a bainha retirada até a abertura do disco direito, no átrio direito; empurrando-se o conjunto, este se ancorou no septo interatrial e liberou o disco esquerdo da veia pulmonar, impactando-se no lado esquerdo do septo (prótese de $30 \mathrm{~mm}$ ).

Em todos os casos, a prótese foi implantada sem dificuldades técnicas. Em cinco pacientes (24\%), houve fluxo residual mínimo, imediatamente após implante, por meio da parte central da prótese, que desapareceu no controle ecocardiográfico prévio à alta hospitalar. Nenhum paciente necessitou de cuidados intensivos, e todos tiveram alta no dia seguinte ao implante. Não houve, na série, mortalidade e nem outras complicações periprocedimento.

Durante o período médio de seguimento, que foi de 10,3 \pm 5 meses, todos os pacientes apresentaram-se livres de sintomas cardiovasculares e, ao exame ecocardiográfico, observou-se ausência de fluxo residual. Em 

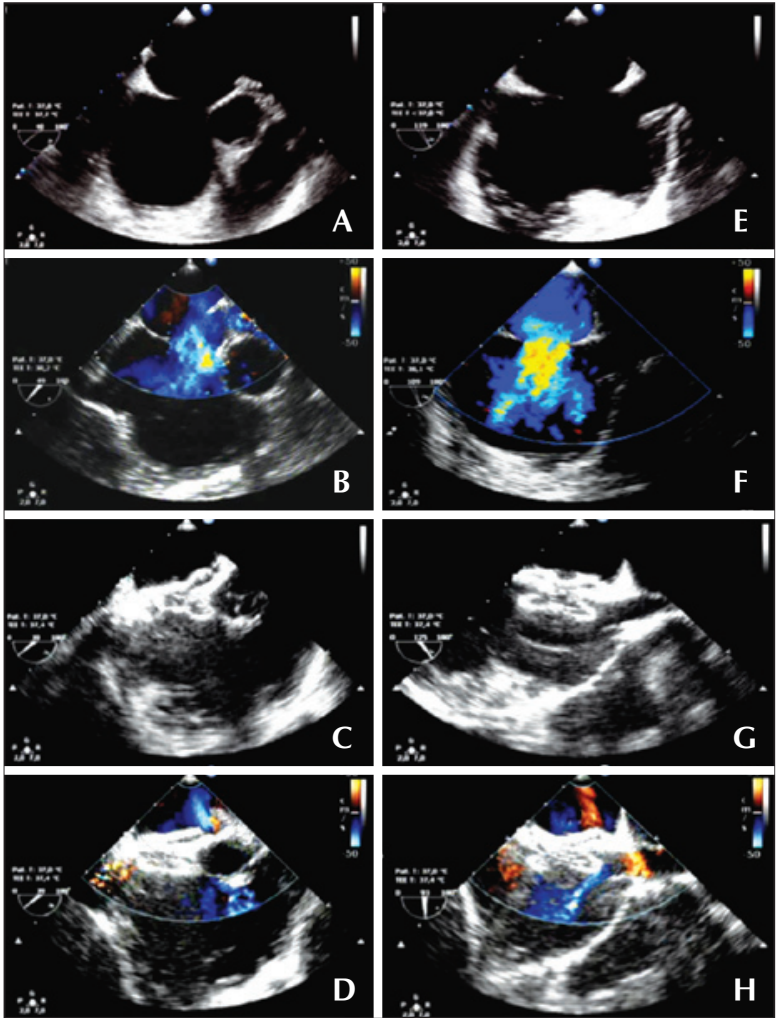

Figura 2. Comunicação interatrial de $16 \mathrm{~mm}$ sem bordo aórtico: eixo curto (A, B, C e D) e eixo longo ou bicaval (E, F, G e H). (A e E) Diâmetro do defeito. (B e F) Fluxo esquerda-direita no color Doppler. (C e G) Posicionamento adequado da prótese, "abraçando" a aorta no eixo curto e os bordos de ambas as cavas no eixo longo. (D e H) Ausência de fluxo residual.

18 casos (86\%), houve normalização do tamanho do ventrículo direito; em 3 pacientes, com idade superior a 50 anos, observou-se persistência de dilatação do ventrículo direito, porém com redução significativa de seu tamanho. Não houve complicações tardias, como erosão, derrame pericárdico, arritmias, formação de trombos ou endocardite infecciosa.

\section{DISCUSSÃO}

A revisão da literatura revelou uma única série publicada com 73 pacientes submetidos à oclusão percutânea de CIA com a utilização dessa prótese, realizada no First People's Hospital of Yanzhou (China). ${ }^{7}$

Na presente série, a taxa de oclusão completa, nas primeiras 24 horas, foi de 100\%. Foi observado, em cinco pacientes, mínimo fluxo residual central através da prótese imediatamente após o implante, o que pode ser explicado por um dos mecanismos de oclusão, que é a trombogenicidade dos retalhos de poliéster, e pelo tempo de coagulação dos pacientes ser variável. Isso se soma ao fato de que, durante o procedimento, é realizada anticoagulação completa. A oclusão completa foi verificada tanto nas comunicações pequenas, quanto nas

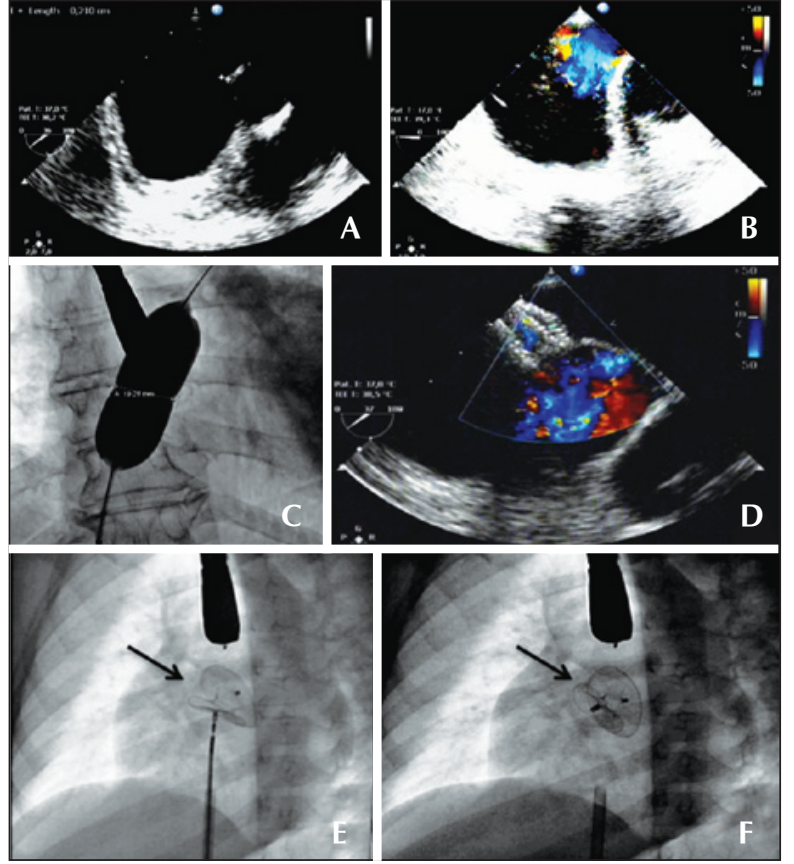

Figura 3. Comunicação interatrial sem bordo aórtico. (A) Ecocardiograma no eixo curto. (B) Color Doppler demonstrando fluxo esquerda-direita. (C) Balão de medição com discreta cintura determinando um diâmetro da comunicação interatrial de 19,5 mm. (D) Color Doppler com o dispositivo implantado e ausência de fluxo residual. (E e F) Fluoroscopia em oblíqua anterior esquerda da prótese implantada, ainda fixa ao cabo de entrega e sob discreta tensão $(C)$ e liberada adotando uma posição mais vertical (D). As setas mostram a maior separação dos discos na parte superior, onde a prótese "abraça" a aorta.

moderadas e grandes, similarmente ao publicado com a utilização de próteses com duplo disco de nitinol. ${ }^{8-13}$

Acredita-se que a ausência de complicações deva-se aos seguintes fatores: flexibilidade da prótese, facilidade do implante e seleção rigorosa dos pacientes. Todos apresentavam características anatômicas ideais para implante com bordos de espessura adequada e tamanho > $5 \mathrm{~mm}$, com exceção do bordo anterossuperior, em que a prótese pode se ancorar, "abraçando" a face posterior da aorta.

O período de seguimento deste estudo ainda é considerado curto para excluir a complicação de maior gravidade e, às vezes, tardia, ou seja, a erosão das paredes atriais com tamponamento cardíaco ou da parede aórtica, com formação de fístula para o átrio esquerdo. ${ }^{14-16}$ Entretanto, as seguintes precauções foram adotadas na seleção do dispositivo para reduzir a probabilidade dessa complicação: evitar o sobredimensionamento da prótese, não implantar um dispositivo com dimensão do disco esquerdo maior que o comprimento máximo do septo interatrial e, ainda, nos casos com bordo anterossuperior deficiente, ancorar o dispositivo "abraçando" a porção posterior da aorta, para que os bordos dos discos, que apresentam maior poder de erosão, não entrem em contato com a justaposição das paredes atrial e aórtica. 


\section{CONCLUSÕES}

A prótese Memopart ${ }^{\circledR}$ apresenta adequada flexibilidade e baixo perfil, sendo de fáceis manuseio e implante. Sua utilização na oclusão percutânea de defeitos septais interatriais do tipo ostium secundum com anatomia favorável mostrou-se um procedimento seguro e eficaz, inclusive em defeitos de grande tamanho, dentro dos limites desta investigação. Uma série maior e com seguimento mais prolongado é necessária para avaliar a eventual ocorrência de complicações tardias.

\section{CONFLITO DE INTERESSES}

Os autores declaram não haver conflito de interesses relacionados a este manuscrito.

\section{AGRADECIMENTOS}

O material Lepu Medical Systems utilizado nos procedimentos foi doado ao HCFMRP-USP pela Valflux Comércio de Materiais Hospitalares (Goiânia, GO), representante no Brasil da Lepu (Shanghai Shape Memory Alloy, Co., Ltd.; Shanghai, China).

\section{REFERÊNCIAS}

1. European Society of Cardiology. ESC Guidelines for the management of grown-up congenital heart disease (new version 2010). Eur Heart J [Internet]. 2010 [cited 2013 Oct 21]; 31:2925-57. Available from: http://eurheartj.oxfordjournals. org/content/31/23/2915.full.pdf

2. Warnes CA, Williams RG, Bashore TM, Child JS, Connolly HM, Dearani JA, et al. ACC/AHA 2008 guidelines for the management of adults with congenital heart disease: a report of the American College of Cardiology/American Heart Association Task Force on Practice Guidelines (Writting Committee to Develop Guidelines on the Management of Adults With Congenital Heart Disease). Developed in Collaboration With the American Society of Echocardiography, Heart Rhythm Society, International Society for Adult Congenital Heart Disease, Society for Cardiovascular Angiography and Interventions, and Society of Thoracic Surgeons. J Am Coll Cardiol. 2008;52(23):e143-263.

3. Masura J, Gavora P, Formanek A, Hijazi ZM. Transcatheter closure of secundum atrial septal defects using the new selfcentering amplatzer septal occluder: initial human experience. Cathet Cardiovasc Diagn. 1997;42(4):388-93.
4. Omeish A, Hijazi ZM. Transcatheter closure of atrial septal defects in children \& adults using the Amplatzer Septal Occluder. J Interven Cardiol. 2001;14(1):37-44.

5. Harper RW, Mottram PM, McGaw DJ. Closure of secundum atrial septal defects with the Amplatzer septal occluder device: techniques and problems. Catheter Cardiovasc Interv. 2002;57(4):508-24.

6. Pinto R, Jain S, Dalvi B. Transcatheter closure of large atrial septal defect in children using the left atrial disc engagementdisengagement technique (LADEDT) - technical considerations and short term results. Catheter Cardiovasc Interv. 2013 Feb 14. [Epub ahead of print]

7. Sun WF, Dong ZF, Gong K, Zhang GP, Cui T, Xia YD, et al. Transcatheter closure with use of the SHSMA occluder in 180 patients with congenital heart defects: preliminary results. Tex Heart Inst J. 2010;37(5):531-7.

8. Du ZD, Hijazi ZM, Kleinman CS, Silverman NH, Larntz K; Amplatzer Investigators. Comparison between transcatheter and surgical closure of secundum atrial septal defect in children and adults: results of a multicenter nonrandomized trial. J Am Coll Cardiol. 2002;39(11):1836-44.

9. Kannan BRJ, Francis E, Sivakumar K, Anil SR, Kumar RK. Transcatheter closure of very large ( $>$ or $=25 \mathrm{~mm}$ ) atrial septal defects using the Amplatzer septal occluder. Catheter Cardiovasc Interv. 2003;59(4):522-7.

10. Pedra CAC, Pedra SRF, Costa RN, Braga SLN, Esteves CA, Fontes VF. Experiência inicial no fechamento percutâneo da comunicação interatrial tipo Óstio Secundum com a prótese Figulla. Rev Bras Cardiol Invasiva. 2010;18(1):81-8.

11. Chamié F, Chamié D, Ramos S, Simões LC, Rossi Filho RI, Tress JC, et al. Oclusão da comunicação interatrial com a nova prótese atriasept-CARDIA: experiência inicial. Rev Bras Cardiol Invasiva. 2009;17(1):91-101.

12. Costa RN, Ribeiro MS, Pereira FL, Pedra SR, Jatene MB, Jatene IB, et al. Percutaneous versus surgical closure of atrial septal defects in children and adolescents. Arq Bras Cardiol. 2013;100(4):347-54.

13. Masura J, Gavora P, Podnar T. Long-term outcome of transcatheter secundum-type atrial septal defect closure using amplatzer septal occluders. J Am Coll Cardiol. 2005; 45(4):505-7.

14. Chessa M, Carminati M, Butera G, Bini RM, Drago M, Rosti L, et al. Early and late complications associated with transcatheter occlusion of secundum atrial septal defect. J Am Coll Cardiol. 2002;39(6):1061-5.

15. Preventza O, Sampath-Kumar S, Wasnick J, Gold JP. Late cardiac perforation following transcatheter atrial septal defect closure. Ann Thorac Surg. 2004;77(4):1435-7.

16. Taggart NW, Dearani JA, Hagler DJ. Late erosion of an amplatzer septal occluder device 6 years after placement. Thorac Cardiovasc Surg. 2011;142(1):221-2. 